\title{
LAPAROSCOPIC ASSISTED ILEOCECAL RESECTION FOR AN APPENDICEAL MUCOCELE WITH 5-YEAR POSTOPERATIVE FOLLOW-UP OF THE PATIENT
}

\author{
SîRBU-BOETI M.P..$^{1,2 * *}$, BĂRBULESCU M. ${ }^{3 \#, ~ H E R L E A ~ V ., 5, ~ L U P E S C U ~ I .2,6 ~ A N D ~ P O P E S C U ~ I .1,2 ~}$ \\ 1Dan Setlacec, General Surgery and Liver Transplantation Center, Fundeni Clinical Institute, Bucharest, Romania. \\ ${ }^{2}$ Carol Davila, University of Medicine and Pharmacy, Bucharest, Romania. \\ ${ }^{3}$ General Surgery Clinic, Prof. Dr. Agrippa lonescu Clinical Emergency Hospital, Bucharest, Romania. \\ ${ }^{4}$ Titu Maiorescu, University of Medicine, Bucharest, Romania. \\ ${ }^{5}$ Department of Pathology, Fundeni Clinical Institute, Bucharest, Romania. \\ ${ }^{6}$ Laboratory of Radiology, Medical Imaging and Interventional Radiology, Fundeni Clinical Institute, Bucharest, Romania. \\ ${ }^{*}$ Corresponding Author: Email- paboet@yahoo.com \\ \#Authors Contributed Equally
}

Received: November 25, 2013; Accepted: December 18, 2013

\begin{abstract}
-
Case Report: One case of appendiceal mucocele (AM) with a large implantation base into the cecum was accurately preoperatively diagnosed by abdomino-pelvic ultrasonography, computed tomography, and colonoscopy. Laparoscopic approach of AM was anticipated for diagnosis confirmation and resection. Due to a large appendiceal base of AM and close proximity of the ileocecal valve to the edge of the mass, visualized by intraoperative ultrasound, the patient was submitted to ileocecal resection. A 6-cm minilaparotomy was used for extraction of the specimen and performance of an extracorporeal ileocolic hand sewn anastomosis. Malignancy was ruled out on frozen sections of the operative specimen. The postoperative patient's evolution was simple, with rapid recovery and short hospitalization. After more than 5 years of follow-up patient remained asymptomatic and disease-free with no malignancy detected.

Conclusion: The limited ileocecal resection using a laparoscopic "no-touch" technique represents a safe treatment for demonstrated benign AM with large base protrusive into cecum in the vicinity of the ileocecal valve.
\end{abstract}

Keywords- appendiceal mucocele, laparoscopic resection, ileocecal resection

Citation: Sîrbu-Boeţi M.P., et al. (2013) Laparoscopic Assisted lleocecal Resection for an Appendiceal Mucocele with 5-Year Postoperative Follow-Up of the Patient. Medical Case Reports, ISSN: 0976-8726 \& E-ISSN: 0976-8734, Volume 4, Issue 1, pp.-49-54.

Copyright: Copyright(02013 Sîrbu-Boeţi M.P., et al. This is an open-access article distributed under the terms of the Creative Commons Attribution License, which permits unrestricted use, distribution and reproduction in any medium, provided the original author and source are credited.

\section{Introduction}

Appendiceal mucocele (AM) is an uncommon lesion recognised as a pathologic entity by Rokitansky in 1842 and formally named by Fere in 1872 [1]. AM is an abnormal aseptic accumulation of mucus in the dilated lumen of the appendix. Recognition of the disease and surgical resection are mandatory because of the potential for malignant transformation and risk of rupture. The pathogenesis of $A M$ is the occlusion of the appendicular lumen from inflammatory strictures, benign or malignant tumors. Four major histological subtypes are distinct: retention cyst, mucosal hyperplasia, cystadenoma, and cystadenocarcinoma [2]. AM is found in $0.07-2.01 \%$ of appendectomy specimens [3, 4]. It might involve even the appendiceal stump after appendectomy [3]. The male to female ratio is $1: 4$ [5]. The patient's average age at the time of diagnosis is 55 years [6]. When symptomatic, AM may give rise to right lower quadrant abdominal pain mimicking acute appendicitis, right lumbar pain due to right extrinsic ureteral obstruction [7], right adnexal [8-10] or cecal tumors [11], obstructive symptoms due to cecocolic intussusception
[12] or intussusception of the mucocele into cecum [13], or gastrointestinal bleeding [14].

The progressive accumulation of mucus eventually results in the blow-out of the appendix and the release of mucus producing cells into the peritoneal cavity. The peritoneal implantation of these mucinous cells results in pseudomyxoma peritonei manifested as progressive abdominal distension with mucinous ascitis. Especially the patients with appendiceal mucinous epithelial neoplasm and particularly mucous adenocarcinoma are prone to develop "jelly belly" syndrome [15]. Hence rupture of the appendiceal wall and spillage of tumor or mucus into the peritoneal cavity must be avoided during surgery. Preoperative diagnosis of AM is helpful in choosing and planning the appropriate surgery.

\section{Case presentation}

A 59-year-old Caucasian woman was admitted to the hospital with a 2 months history of intermittent abdominal pain in the right inferior quadrant of the abdomen. The patient had laparoscopic cholecys- 
tectomy for gallbladder lithiasis 12 years ago. Standard laboratory tests were within normal ranges. Serum levels of AFP $(1.87 \mathrm{ng} / \mathrm{ml}$, NR 0-15), CA 19-9 (41.47 Ul/ml, NR 0-37), CEA (2.35 ng/ml, NR 05) were within normal ranges. Abdominal ultrasonography detected a cystic mass situated in the right inferior quadrant. Abdominopelvic enhanced computed tomography (CT) with oral contrast substance showed a $10 \times 5 \mathrm{~cm}$ cystic lesion bulging into the cecal lumen [Fig-1]. Colonoscopy demonstrated a protuberant submucosal soft mass at the base of the cecum covered with normal mucosa and centered by the appendiceal orifice [Fig-2]. With a high suspicion of AM with intracecal extension biopsy of the mass was prohibited, laparoscopic exploration was decided, and an ileocecal resection anticipated.

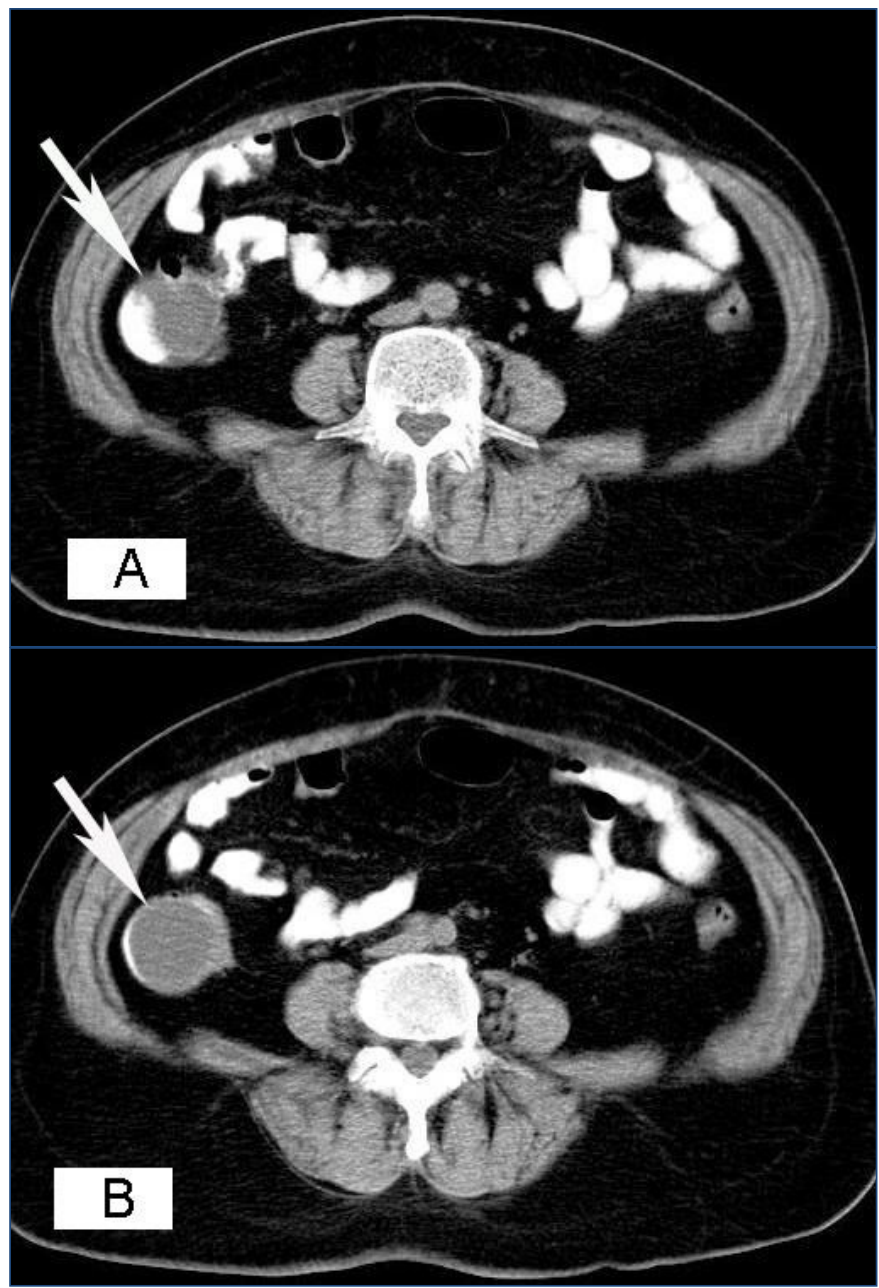

Fig. 1- Abdominal CT with positive oral contrast at the level of the ileocecal valve $(\mathbf{A})$ and below the ileoceal valve $(B)$ - protrusive cystic mass (marked with a white arrow) located in the cecum involving the ileocecal valve.

The patient was placed in supine position with the first surgeon and cameraman standing on the left side. Four trocars were inserted as follows: a supraumbilical 10-mm diameter trocar for camera, a 5$\mathrm{mm}$ epigastric trocar for atraumatic grasper forceps, a suprapubic 10-mm and left sided 12-mm trocar for atraumatic grasper forceps, laparoscopic ultrasound probe, Ligasure forceps, and linear Endo GIATM stapler [Fig-3].

On laparoscopic exploration the appendix was found enlarged, with smooth intact serosa and slightly dilated capillaries, with its base impinging the cecum in the proximity of the ileocecal valve [Fig-4].

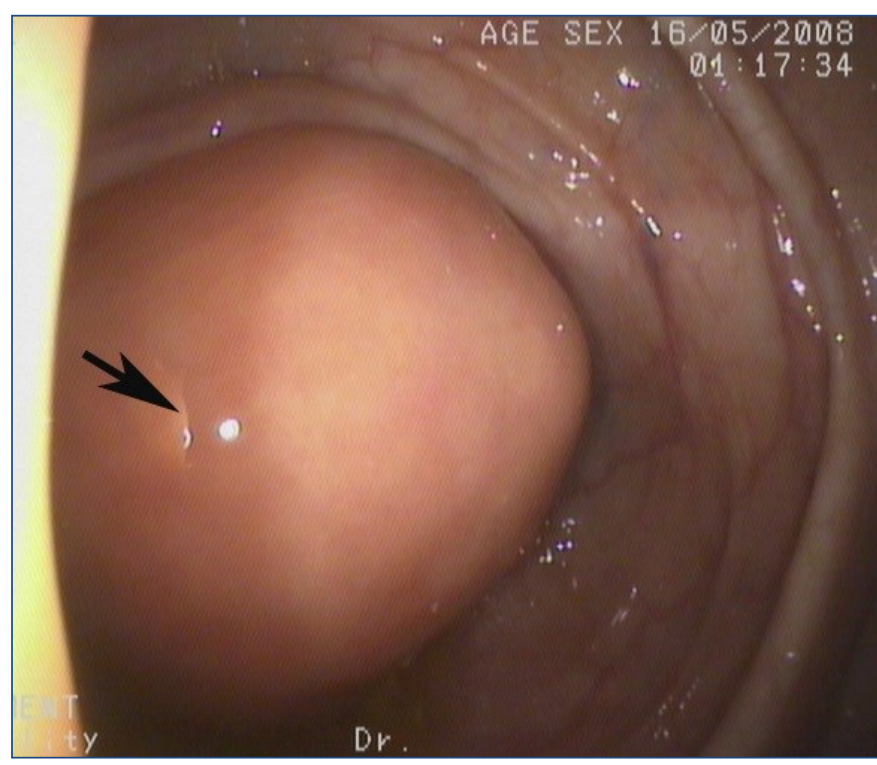

Fig. 2- Preoperative colonoscopic aspect of appendiceal mucocele: submucosal cecal mass bulging into the cecal lumen and centered by appendiceal orifice (black arrow) - "volcano sign".

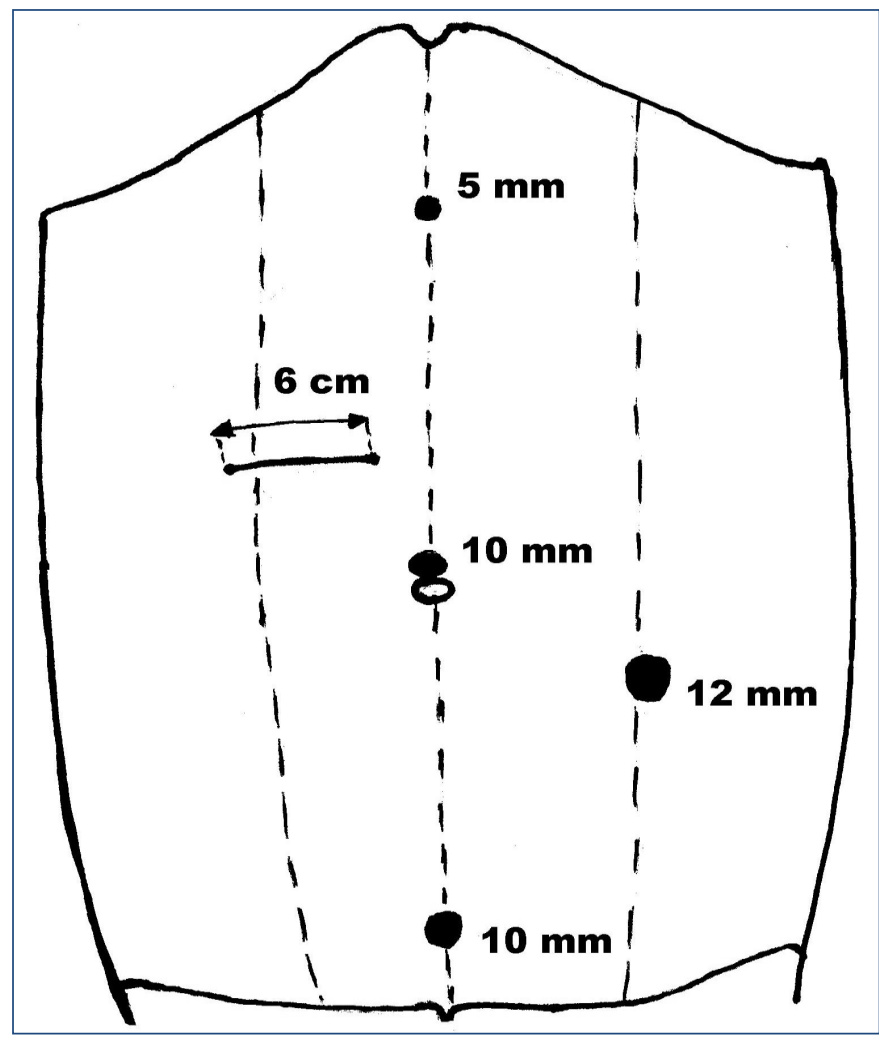

Fig. 3- Disposition of trocars used for laparoscopic exploration and ileocecal resection with the $6-\mathrm{cm}$ extraction minilaparotomy site used for specimen extraction and extracorporeal anastomosis.

Mild visceral adhesions around the cecum were encountered and dissected, sparing the cecal appendix. The peritoneum was normal. After initial laparoscopic exploration, laparoscopic ultrasound examination was performed using a flexible linear probe for ruling out loco-regional lymph node involvement, identification of the vascular pedicles, and delineation of the ileocolic resection [Fig-4]. Laparoscopic ileocecal resection including the cecal appendix and mesoappendix was performed using the "no-touch isolation tech- 
nique", carefully avoiding direct manipulation of the AM. The instrumental manipulation was done non-traumatically on the healthy tissues of the colon and ileum without touching the mucocele. Therefore the operation was completed with no injury or spillage of the AM. A 45-mm, thick-tissue Endo GIATM linear stapler was used for laparoscopic transection of the last ileal loop and proximal half of the ascending colon [Fig-5, Fig-6]. A 6-cm minilaparatomy on the right side and slightly above the umbilicus was performed for the specimen removal within a non-permeable bag [Fig-3]. The specimen was sent for frozen section examination. Macroscopically, the appendix was found dilated up to $8 \mathrm{~cm}$ diameter, occupied by a jelly -like mucoid mass [Fig-7], and microscopically covered with high cylindric epithelium with normochrome basal nuclei and no signs of malignancy found [Fig-8]. With the benign confirmation of AM the operation was carried on. Both ends of the bowel were exteriorised through the minilaparotomy [Fig-9]. The stapled lines of both ileum and ascending colon were enforced using one 4.0 PDS seromuscular continuous suture. A side-to-side ileocolic anastomosis was fashioned using two layers of continuous 4.0 PDS. The anastomosis was repositioned intraperitoneally. The peritoneal cavity was checked for bleeding. No peritoneal lavage was used. One drainage tube was left in the pouch of Douglas and removed 48 hours later. The postoperative course was uneventful, with first bowel movement on the 3rd postoperative day. The patient was discharged on the 4th postoperative day.

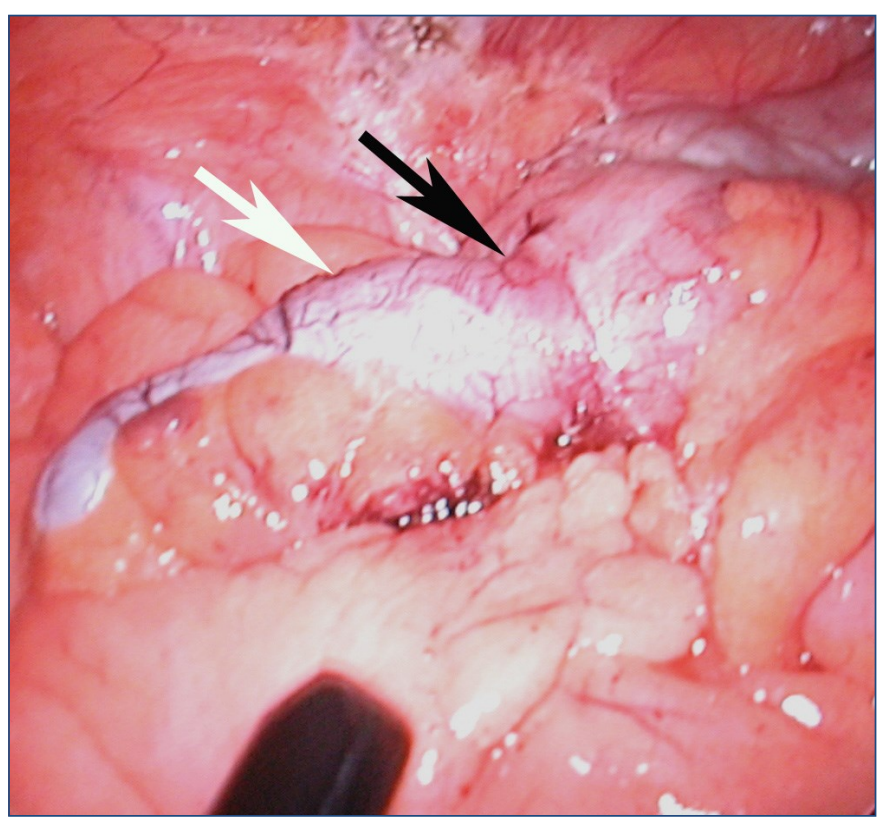

Fig. 4- Intraoperative laparoscopic and ultrasound exploration of the appendiceal tumor (white arrow) with its base bulging into the cecum lumen (black arrow).

Paraffin section examination of the specimen confirmed the diagnosis of simple AM with no signs of malignancy [Fig-8]. No other postoperative treatment was necessary.

A follow-up was recommended to the patient. Serum tumour markers (CEA and CA 19.9) were checked every 6 months, colonoscopy and computed tomography were performed annually. The results were normal.

At 62 months postoperatively the patient is disease- and symptomfree, with a normal 5-year control colonoscopy[Fig-10]. The bowel movement is normal with 1-2 formed stools daily.

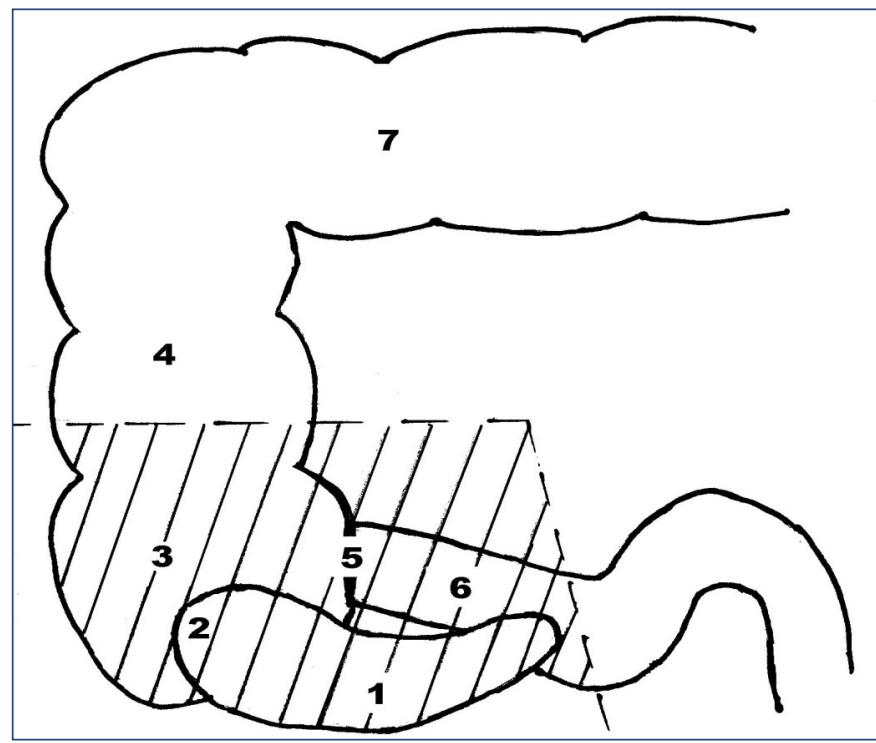

Fig. 5- Before resection schematic illustration: 1. tumor of the appendix (mucocele) placed toward the ileum; 2. base of the mucocele implanted into the cecum; 3. cecum; 4. ascending colon; 5. ileocecal valve; 6 . last ileal loop; 7. transverse colon.

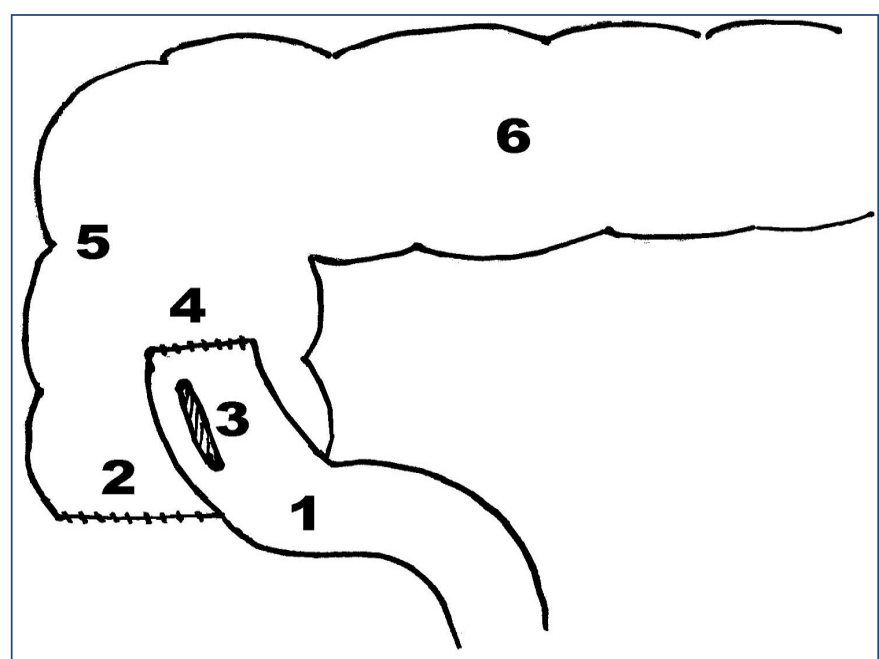

Fig. 6- After resection schematic illustration: 1. last ileal loop; 2. stapled inferior edge of the ascending colon; 3. isoperistaltic side-to -side ileocolic anastomosis (extra corporeally performed); 4. proximal stapled end of the terminal ileum stump; 5. ascending colon; 6. transverse colon

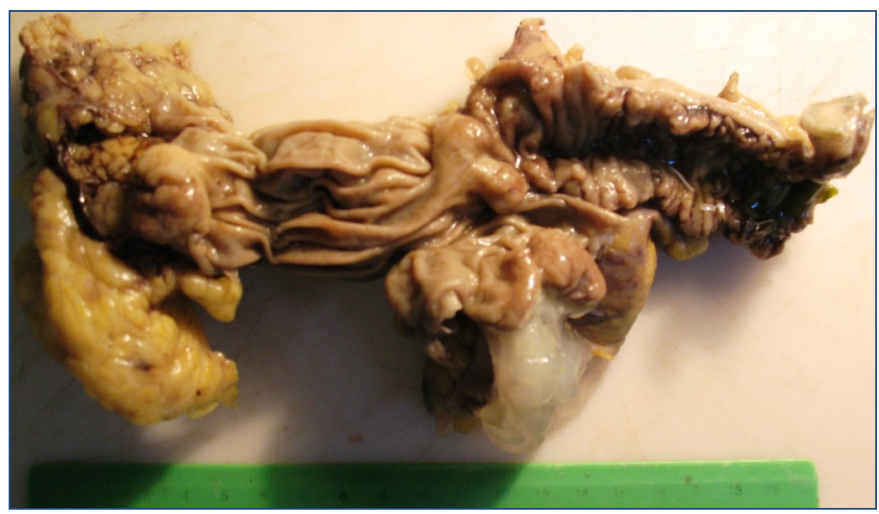

Fig. 7- Resected specimen with jelly-like white mucus content of the appendiceal sectioned tumor. 


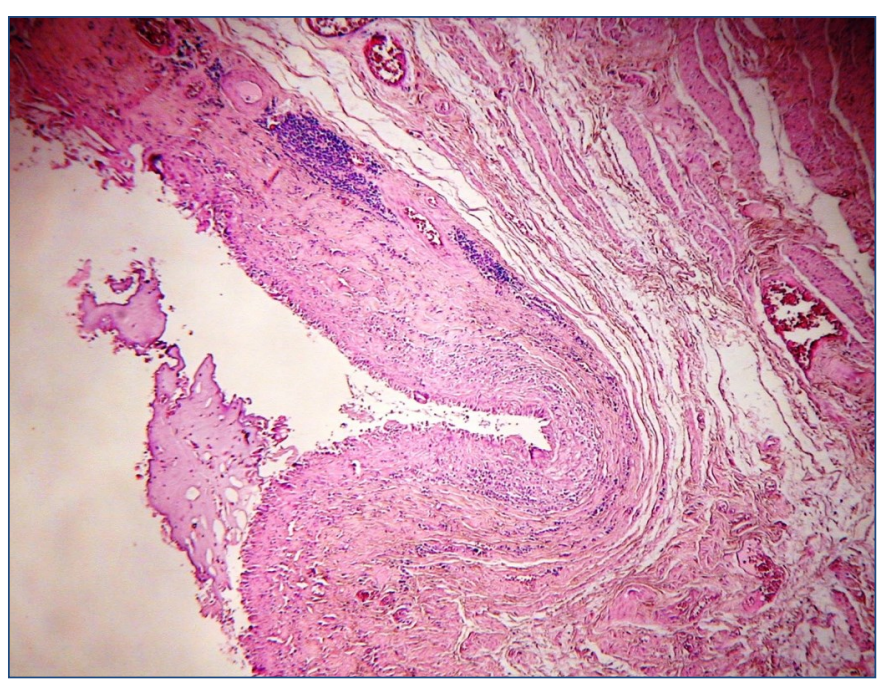

Fig. 8- Microscopical paraffin section examination of the appendiceal tumor showing a benign mucocele (Hematoxillin \& Eosin staining, x40).

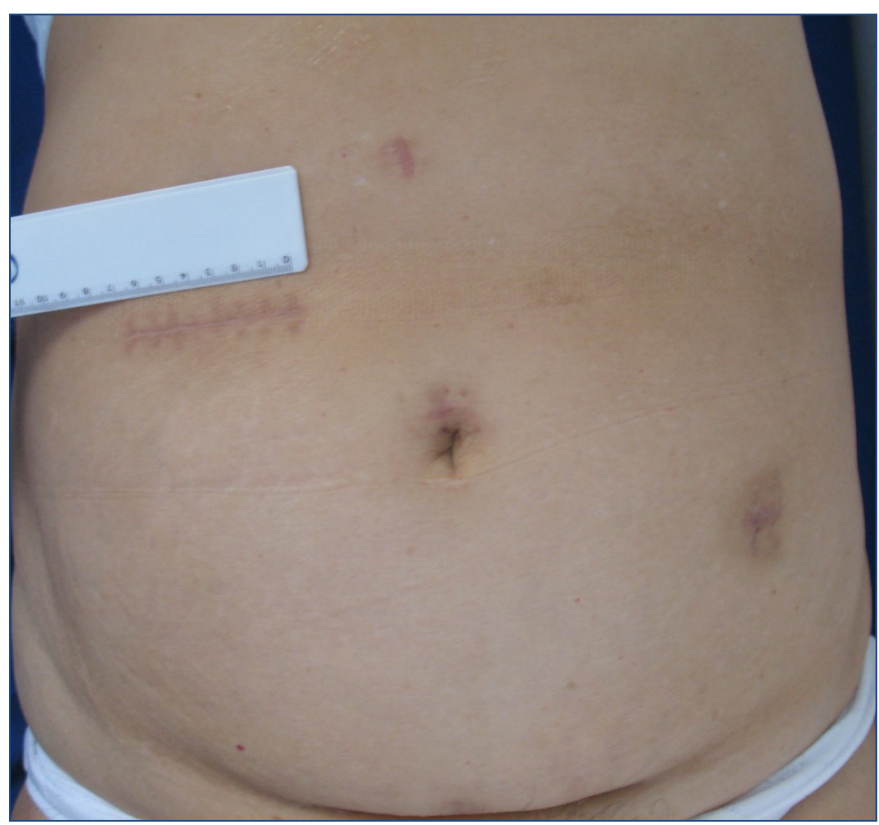

Fig. 9- Abdominal scars one-month after surgery with the 6-cm length minilaparotomy site (white ruler).

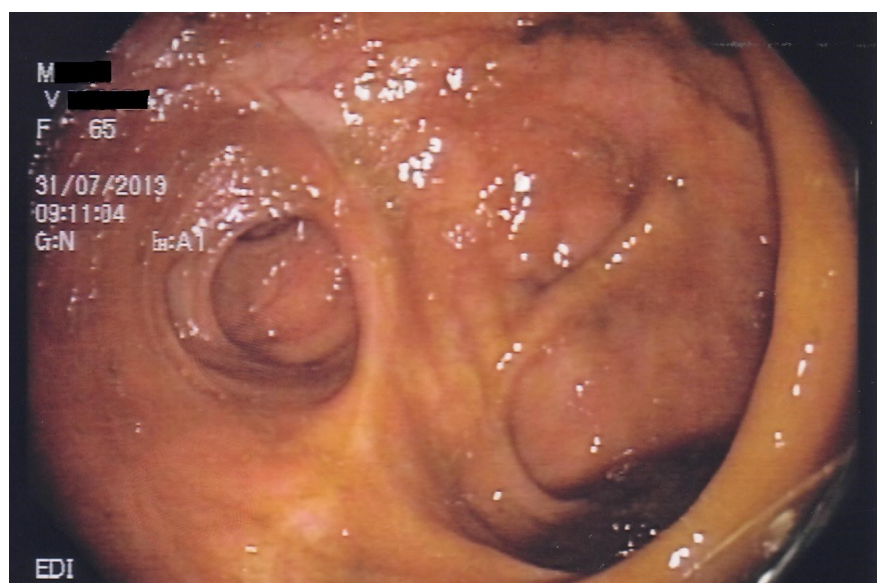

Fig. 10- Colonoscopic normal aspect of the side-to-side ileocolic anastomosis at 5-year postoperative check-up.

\section{Discussions}

$\mathrm{AM}$ is a rare entity with nonspecific preoperative presentation. A significant proportion of all AMs are discovered incidentally at abdominal imaging, colonoscopy, or unrelated abdominal surgery. It is reported more commonly in middle-aged women with right lower quadrant pain as the main complain. Clinical manifestations of AM are often indistinguishable from those of acute appendicitis. In most cases AM is intraoperatively diagnosed. However, a correct preoperative diagnosis is possible based on specific imagistic and/or colonoscopic patterns. Ultrasound remains a real-time, efficient diagnostic tool for immediate assessment of patients with acute abdominal pain. Different sonographic findings of AM have been described previously: a cystic structure with anechoic fluid, un elongated hypoechoic mass with fine internal echoes, appendiceal wall calcification, and, when AM is complicated with pseudomyxoma peritonei, scalloping of the hepatic contour or ascites. The onion skin sign is consider pathognomonic for AM [10]. Appendiceal outer diameter can be a good predictor for accurate preoperative diagnosis. An outer diameter threshold of $6 \mathrm{~mm}$ has been established for acute appendicitis diagnosis. An appendiceal threshold diameter of $15 \mathrm{~mm}$ was the best for AM, with a sensitivity of $83 \%$ and specificity of $92 \%$ [5]. Larger lesions are suggestive of mucinous cystadenoma or cystadenocarcinoma [5]. The classical appearance of AM on colonoscopy is the "volcano sign", with the appendiceal orifice seen at the center of a typical bulbous submucosal cecal lesion [16-18]. Biopsies are forbidden due to the risk of spillage and peritoneal seeding. Colonoscopy is necessary in all patients with AM to exclude synchronous colon tumors [19-22].

All AMs must be managed by surgical resection. Because the endoscopic and imaging appearances are unable to differentiate benign from malignant lesions, a frozen section examination of the operative specimen is mandatory.

In case of an emergency mimicking acute appendicitis, bowel obstruction, or organ torsion, preoperative diagnosis of the AM could be missed. Laparoscopic exploration represents an excellent diagnostic tool especially when it is performed with urgency and thorough preoperative examinations are not run [23]. Moreover it allows a complete abdominal exploration to rule out other tumors, like colon and ovaries, that are known to be associated with AM [24]. Laparoscopic ultrasound examination contributes to ruling out locoregional lymph nodes and assisting the delineation of cecal resection.

Any suspicion of AM should oblige to an appropriately planned surgical approach. The main goals of subsequent surgery are the complete removal of the AM and avoidance of peritoneal dissemination.

The decision to pursue laparoscopic resection of AM depends on localization and accessibility to AM and surgeon's laparoscopic experience. Some authors consider AM to be a contraindication for laparoscopic appendicectomy $[25,26]$. We concur with other authors that laparoscopic approach offers a higher visibility and larger space for visceral manipulation in a no-touch technique $[6,21,23$, $27,28]$. Moreover, due to avoidance of a large incision, it is followed by a rapid recovery and better cosmetic outcome.

If there is no evidence of synchronous tumor, peritoneal spillage, dilated appendiceal base, invasion or positive sentinel lymph nodes, appendicectomy remains the definitive adequate treatment for benign AM [22]. If malignant disease is demonstrated, right hemicolectomy is the standard. If any suspicion of rupture of AM exists, lapa- 
roscopy should be avoided and a midline abdominal incision is suggested [26]. If the base of the appendix is larger than $2 \mathrm{~cm}$, a partial cecum resection including appendicectomy is appropriate $[2,29$, 30]. If intussusception of AM is found, an ileocecal resection is indicated $[13,31]$. We consider that for benign AMs with a dilated implantation base of appendix into cecum, right hemicolectomy is not justified as it was recommended by other authors [32]. Instead, these AMs must be submitted to cecal or ileocecal resection. A partial cecal resection can be performed with Endo GIATM stapler if the line of section spares the ileocecal valve. Due to the close vicinity of the ileocecal valve to AM involving the base of cecum, as it was encountered in our case, a limited stapled cecum resection is not technically possible. In such cases, a laparoscopic ileocecal resection in block with the cecal appendix and mesoappendix represents the best solution [33]. The ileocecal resection is followed by intracorporeal or extracorporeal ileocecal anastomosis. We chose to perform an extracorporeal manual ileocecal anastomosis through a small parietal incision placed in accordance with the colonic segment. Because of a high lying cecum an upper right horizontal minilaparotomy was used. Thus, a laborious operation through the classical McBurney incision could be avoided.

A minilaparotomy has the advantage of a facile extraction of the resected specimen. An extraction bag must be used to avoid direct contact with the abdominal wall and thus eliminate the risk of AM's rupture.

The specimen should be examined on cryosections to rule out malignancy. When pathological diagnosis is not possible at the time of surgery, knowing that only $10-20 \%$ of AM are malignant, right hemicolectomy should not be performed [30]. This surgical procedure will be further needed if malignancy is diagnosed on the removed appendix.

Simple and hyperplastic AM do not require follow-up, benign mucocele should be followed-up as an adenomatous polyp with colonoscopy, and the malignant one should be followed-up as a colonic adenocarcinoma, with CEA level and serial colonoscopies [30]. Being patients at high-risk of cancer development, we recommend the screening of the tumor markers every 6 months even when a simple or benign AM is documented.

\section{Conclusions}

AM can be accurately diagnosed preoperatively by abdomino-pelvic ultrasonography, CT, and colonoscopy. The treatment of $A M$ is always surgical and depends on the integrity and size of the appendix base and the histological type of the original lesion on the frozen sections. Laparoscopic approach of AM must be carefully planned ahead for diagnosis confirmation, followed or not by laparoscopic resection. Laparoscopic ultrasound examination aids in surgical resection by visualization of the AM's border. If a large appendiceal base is encountered in close proximity to the ileocecal valve, the patient should be submitted to ileocecal resection. A minilaparotomy can be used for specimen extraction and extracorporeal ileocecal anastomosis. Malignancy must be ruled out on frozen sections. A follow-up including colonoscopy and tumor markers is recommended even for benign AM. Laparoscopic ileocecal resection is a feasible and safe therapeutic procedure for benign AM protruding into the base of cecum, with excellent short- and long-term outcomes. It should be performed when a simple laparoscopic appendectomy with cecectomy is not technically possible and right hemicolectomy is not justified.

\section{Conflict of Interest}

Drs. Sîrbu-Boeţi M.P., Bărbulescu M., Herlea V., Lupescu I. and Popescu I. have no conflicts of interest or financial ties to disclose.

\section{References}

[1] Kahn M., Friedman I.H. (1979) Dis. Colon. Rectum., 22(4), 267269.

[2] Caracappa D., Gulla N., Gentile D., Listorti C., Boselli C., Cirocchi R., Bellezza G., Nova G. (2011) Ann. Ital. Chir., 82(3), 239-245.

[3] Lien W.C., Liu K.L., Kuo M.Y., Fang C.C., Lin M.T., Wang H.P., Chen W.J. (2004) Surgery, 136(1), 93-94.

[4] Karakaya K., Barut F., Emre A.U., Ucan H.B., Cakmak G.K., Irkorucu O., Tascilar O., Ustundag Y., Comert M. (2008) World J. Gastroenterol., 14(14), 2280-2283.

[5] Lien W.C., Huang S.P., Chi C.L., Liu K.L., Lin M.T., Lai T.I., Liu Y.P., Wang H.P. (2006) Am. J. Emerg. Med., 24(7), 801-805.

[6] Huang C.C., Lo L.K., Koh L.W. (1994) J. Am. Assoc. Gynecol. Laparosc., 1(2), 174-176.

[7] De Pablo Cárdenas A., Lozano Uruñuela F., Pinós Paul M.A., Jiménez Aristu J.I., Jiménez Calvo J.M., Ruiz Ramo M, Guarch Trovas R., Santiago González de Garibay A.M., Sebastián Borruel J.L. (2001) Arch. Esp. Urol., 54(5), 451-454.

[8] Balci O., Ozdemir S., Mahmoud A.S. (2009) Taiwan J. Obstet. Gynecol., 48(4), 412-414.

[9] Pureti M., Hollingworth J., Palaniappan M. (2006) J. Obstet. Gynaecol., 26(5), 484-485.

[10]Bartlett C., Manoharan M., Jackson A. (2007) J. Med. Case Rep., 19(1), 183-185.

[11]Jiménez Mazure C., Ramírez Plaza C.P., Valle Carbajo M., Santoyo Santoyo J. (2009) Rev. Esp. Enferm. Dig., 101(6), 447449.

[12]Coulier B., Pestieau S., Hamels J., Lefebvre Y. (2002) Eur. Radiol., 12(2), 324-328.

[13]Tsunoda T., Eto T., Maeda H., Mochinaga N., Terada M., Matsuo S., Tsuchiya R., Takahara Y. (1992) Surg. Today, 22(5), 470-473.

[14]Hayashi T., Kawahara H., Yoshimoto K., Kashiwagi H., Yanaga K., Komine K. (2008) Int. J. Surg., 6(6), e15-e17.

[15]Smeenk R.M., van Velthuysen M.L., Verwaal V.J., Zoetmulder F.A. (2008) Eur. J. Surg. Oncol., 34(2), 196-201.

[16]Hamilton D.L., Stormont J.M. (1989) Gastrointest Endosc., 35 (5), 453-456.

[17]Raijman I., Leong S., Hassaram S., Marcon N.E. (1994) Endoscopy, 26(3), 326-328.

[18]Zanati S.A., Martin J.A., Baker J.P., Streutker C.J., Marcon N.E. (2005) Gastrointest. Endosc., 62(3), 452-456.

[19]Debray C., Leger L., de Saint-Maur P. (1973) Sem. Hop., 49 (18), 1290-1292.

[20]Fujiwara T., Hizuta A., Iwagaki H., Matsuno T., Hamada M., Tanaka N., Orita K. (1996) Dis. Colon. Rectum., 39(2), 232-236.

[21]Stang A., Braumann D., Teichmann W. (2004) Dtsch. Med. Wochenschr., 129(43), 2295-2298.

[22]Kim-Fuchs C., Kuruvilla Y.C., Angst E., Weimann R., Gloor B., 
Candinas D. (2011) Case Rep. Gastroenterol., 5(3), 516-522.

[23]Miraliakbari R., Chapman W.H. (1999) J. Laparoendosc. Adv. Surg. Tech., 9(2), 159-163.

[24]Roberge R.J., Park A.J. (2006) J. Emerg. Med., 30(3), 303-306.

[25]Gonzá.lez-Moreno S., Shmookler B.M., Sugarbaker P.H. (1998) Surg. Endosc., 12(9), 1177-1179.

[26]Dhage-Ivatury S., Sugarbaker P.H. (2006) J. Am. Coll. Surg., 202(4), 680-684.

[27]Lau H., Yuen W.K., Loong F., Lee F. (2002) Surg. Laparosc. Endosc. Percutan. Tech., 12(5), 367-370.

[28]Gortchev G., Tomov S., Dimitrov D., Nanev V., Betova T. (2010) Obstet. Gynecol. Int., 281053, doi: 10.1155/2010/28105

[29]Minagawa M., Ishikawa H., Date K., Kosugi S., Hatakeyama K., Endo K., Kimura K., Fukuda F. (2001) Gastrointest. Endosc., 53 (4), 493.

[30]de Abreu Filho J.G., de Lira E.F. (2011) J. Coloproctol. (Rio J.), 31(3), 276-284.

[31]Huang H.C., Liu T.P., Jeng K.S. (1994) Zhonghua Yi Xue Za Zhi (Taipei), 53(2), 120-123.

[32]Huang C.C., Lo L.K., Koh L.W. (1994) J. Am. Assoc. Gynecol. Laparosc., 1(2), 174-176.

[33]Simone M., Marescaux J. (2006) Laparoscopic ileo-caecal resection for appendicular mucocele. 